\title{
Smartphones y tablets en familia. Diseño de un instrumento diagnóstico
}

\author{
Irina Salcines-Talledo $\left({ }^{*}\right)$, Antonia Ramírez-García ${ }^{* *}$ y Natalia González-Fernández (*) \\ $\left.{ }^{*}\right)$ Universidad de Cantabria - España $\left(^{* *}\right)$ Universidad de Córdoba - España
}

\begin{abstract}
RESUMEN
La repercusión de los dispositivos móviles, especialmente las Tablets y los Smartphones, en todas las esferas sociales es incuestionable. En este artículo se muestra el diseño, validación y aplicación piloto de un cuestionario dirigido a las familias españolas, con la finalidad de recoger información sobre la influencia de los dispositivos ya mencionados, en las dinámicas familiares. Concretamente, sobre el conocimiento que poseen los padres, su influencia en la comunicación familiar, las rutinas y hábitos que se generan, la normativa, mediación y necesidades formativas al respecto. Se presenta el procedimiento de diseño, validación y aplicación del cuestionario, para comprobar su validez de contenido, la fiabilidad y el comportamiento del mismo. Los resultados demuestran que la herramienta de evaluación diagnóstica es válida, fiable y recoge con precisión los datos sobre las variables que lo configuran.
\end{abstract}

Palabras Clave: Tablets, Smartphones, Tecnologías de la Comunicación y la Información, Familia, Cuestionario.

\section{Smartphones and tablets in family. Design of a diagnostic instrument}

\section{ABSTRACT}

The impact of mobile devices, especially Tablets and Smartphones, in all social spheres is unquestionable. This paper shows the design, validation and pilot application of a questionnaire to Spanish families. Its purpose is to collect information about the influence of these devices on family dynamics. Specifically, about the knowledge that parents have, their influence on family communication, routines and habits that are generated, regulations, mediation and training needs in this regard. The procedure of design, validation and application of the questionnaire is presented to check its content validity, reliability and behavior. Results show that this tool diagnostic is valid, reliable and accurately collect data on variables that make it up.

Keywords: Tablets, Smartphones, Technology of the Information and Communication, Family, Questionaire.

\section{Agradecimientos}

Este trabajo se ha elaborado en el marco de Alfamed (Red Interuniversitaria Euroamericana de Investigación en Competencias Mediáticas para la Ciudadanía), con el apoyo del Proyecto I+D+I Coordinado “Competencias mediáticas de la ciudadanía en medios digitales emergentes (Smartphones y Tablets): practicas innovadoras y estrategias educomunicativas en contextos múltiples" (EDU2015-64015-C3-1-R) (MINECO/FEDER), y de la "Red de Educación Mediática" del Programa Estatal de Investigación Científica-Técnica de Excelencia, Subprograma Estatal de Generación de Conocimiento (EDU2016-81772-REDT), financiados por el Fondo Europeo de Desarrollo Regional (FEDER) y Ministerio de Economía y Competitividad de España.

\section{Introducción}

El conocimiento y uso de las Tecnologías de la Información y la Comunicación (TIC) en la sociedad actual, es cada vez mayor gracias a las oportunidades y posibilidades que ofrecen tanto en el ámbito privado como profesional. La sociedad hiperconectada está consolidando la banda ancha del 3G en los países de la OCDE, en particular en la Unión Europea (Ramos y Ortega-Mohedado, 2017). Igualmente, cada vez se requiere de dispositivos móviles más versátiles y potentes, como son el Smartphone y la Tablet, que están cambiando el mundo de las comunicaciones y las costumbres de los navegantes digitales de los medios, los digital-digito-nautas (Ortega, González y Pérez, 2015).

Según el Instituto Nacional de Estadística (2017), el 69,1\% de los menores españoles comprendidos entre 10 y 15 disponen de un Smartphone propio. El porcentaje aumenta hasta un $94 \%$ en la población de 15 años. En la misma línea, el Informe Ditrendia (2017) señala que, a nivel mundial, los usuarios utilizan el Smartphone una media de 170 minutos al día y la Tablet 75 minutos, siendo el teléfono móvil el dispositivo más utilizado por los españoles para acceder a internet (94,6\%), por otro lado, el 61,3\% de internautas utiliza la Tablet todos o casi todos los días.

Resulta interesante, completar la información anterior con los datos recogidos por la Fundación Telefónica (2017) sobre la motivación fundamental de los usuarios para conectarse a In- 
ternet. En este caso, el ocio sigue siendo la motivación principal $(35,5 \%)$ frente a un $31,7 \%$ de los internautas cuya finalizad es comunicarse con familiares y amigos, y un $28,6 \%$ que lo hace por motivos profesionales, educativos y de productividad. Por último, hay un gran incremento $(61,9 \%)$ en los jóvenes entre catorce y diecinueve años que se conectan con finalidades educativas.

Ante esta realidad, nos preguntamos cómo influye la inclusión de los dispositivos digitales en los hogares y en la comunicación familiar, si ésta es fluida y profunda o, estamos ante el riesgo de aislamiento entre los miembros de la familia, por la ausencia de normas internas respecto a su uso.

Autores como Prensky (2009), consideran que, pese a los riesgos de la evolución tecnológica global, es necesario desarrollar la competencia para utilizar las tecnologías y hacerlo de manera adecuada, con el objetivo de mejorar capacidades cognitivas innatas, como el proceso de toma de decisiones prácticas, creativas y de contenido apropiado para la resolución de problemas humanos y, al logro de tal competencia, lo denomina sabiduría digital.

Los estudios realizados sobre la influencia de la tecnología en la educación y la comunicación en la esfera privada han sido múltiples (De la Villa y Suárez, 2016; Dryāgan, 2010; Fombona y Roza, 2016; Martínez, Enciso y González Castillo 2015; Pedrero, Rodríguez y Ruiz, 2012) y, cada uno de ellos ha evidenciado cuestiones interesantes sobre el problema. El nuevo informe $\mathrm{Di}$ gital in 2017 (2017), revela que más de la mitad de la población mundial usa Internet, un dato relevante refleja que 1 de cada 5 personas en el mundo ya compra online y, que el Smartphone es el dispositivo más utilizado para acceder a las redes sociales y a contenidos audiovisuales. En el ámbito familiar Lepicnik y Samec (2013) afirman que la mayoría de los niños menores de cuatro años, viven en un entorno tecnológico en el que sus familias apoyan el aprendizaje mediante TIC, aun reconociendo como consecuencia negativa, la pérdida del contacto con la realidad e incluso la adicción.

De ahí, la necesidad de conocer las dinámicas familiares ante la responsabilidad de educar en un uso razonable y saludable de los dispositivos móviles, manteniendo una comunicación de calidad permanente entre los miembros de la familia, siendo sensibles a las necesidades del otro y, estableciendo normas claras y firmes.

Si bien hay diversos cuestionarios y escalas de medida, que recolectan datos relacionados con los dispositivos móviles, como por ejemplo el uso pedagógico o educativo que se hace de los mismos (Henríquez, González y Organista, 2014; Merino, Cabello y Merino, 2017; Salcines-Talledo y González-Fernández, $\underline{2015,2016)}$, la dependencia y adicción que puede generar su uso
(Bianchi y Phillips, 2005; Billieux, Van der Linden y Rochat, 2008; Jenaro, Flores, Gómez-Vela, González-Gil y Caballo, 2007; Kawasaki et al., 2006; Toda, Monden, Kubo y Morimoto, 2006; Yang, Yen, Ko, Cheng y Yen, 2010), o los usos inapropiados de los dispositivos móviles por los adolescentes (Álvarez-García, Dobarro y Núñez, 2015; Rodríguez-Gómez, Castro y Meneses, 2018), son menos las aportaciones relacionadas con su repercusión en el contexto familiar (Stein, Lawrence y Greenberg, 2016; Symons, Ponnet, Emmery, Walrave y Heirman, 2017; Tanis, Louw y Buijzen, 2017; Tsai, Chang y Ho, 2016) y, no aparecen instrumentos específicos para su abordaje. El objetivo de este artículo es presentar el proceso seguido de validación y aplicación piloto de un instrumento de evaluación diagnóstica, que permita recabar datos sobre el impacto de los dispositivos móviles en el ámbito familiar.

\section{Metodología}

\section{Procedimiento}

Para la validación del cuestionario se han realizado dos tipos de análisis. En primer lugar, se analizó la validez de contenido de la herramienta mediante un panel de expertos a través de la cumplimentación por 8 jueces expertos de una plantilla de valoración. Y, en segundo lugar, se utilizó el análisis de la consistencia interna (Alfa de Cronbach) para el estudio de la fiabilidad. Posteriormente, se han analizado algunos de los datos más destacables de la aplicación piloto del cuestionario a través de estadística descriptiva.

\section{Muestra}

Se procedió a la aplicación del cuestionario en una muestra piloto durante el mes de agosto de 2017 para el cálculo de la fiabilidad y el análisis del comportamiento del cuestionario. El número total de personas encuestadas fue de 40 siguiendo las recomendaciones de Gil-Pascual (2016), que señala como número adecuado para las aplicaciones piloto entre 25 y 50 personas.

A través de la aplicación de WhatsApp, se envió el enlace a la aplicación online del cuestionario a 65 personas, y fueron 40 las que finalmente contestaron y formaron parte de la muestra.

A continuación se presenta la tabla 1 con los principales datos identificativos de los participantes. Puesto que algunas de las preguntas estaban condicionadas a cuestiones previas, y la muestra que respondió no cumplía el criterio propuesto por Gil-Pascual (2016) se ha realizado una selección de los ítems que contaban con un mínimo de 25 respuestas.

Tabla 1. Datos identificativos de los participantes en la aplicación piloto

\section{CARACTERÍSTICAS}

Sexo:

- Hombre

- Mujer

Edad:

- Menos de 25 años

- Entre 26 y 34 años

- Entre 35 y 44 años

- Entre 45 y 60 años

- Más de 60 años
PORCENTAJE

CARACTERÍSTICAS

PORCENTAJE

Número de personas en el hogar:

$17,5 \%$

$82,5 \%$

- 2

$\cdot 3$

$\cdot 5$

$17,5 \%$

$30,0 \%$

$40,0 \%$

$12,5 \%$

Número hijos en edad escolar:

- 0

$15,8 \%$

$26,3 \%$

$5,0 \%$

$25,0 \%$

$65,0 \%$

$2,5 \%$
$47,4 \%$

$10,5 \%$ 


\section{CARACTERÍSTICAS}

PORCENTAJE

CARACTERÍSTICAS

PORCENTAJE

Procedencia:

- Barcelona

- Cantabria

- Córdoba

- Madrid

- Málaga

- Vizcaya

Vive en un contexto:

- Rural

- Urbano

Hijos:

- Sí

$\cdot$ No

Convive con su pareja:

- Sí

$\cdot$ No

Rol respecto a los menores del hogar:

- Progenitor1/Padre

- Progenitor2/Madre

- Otro

Modalidad familiar:

- Nuclear

- Monoparental

- Familia reconstituida

- Familia extensa
Ocupación laboral del padre:

- Empleado público/Funcionario 36,7\%

- Autónomo 10,0\%

- Trabajador por cuenta ajena $\quad 30,0 \%$

- Desempleado $\quad 10,0 \%$

· Jubilado $\quad 13,3 \%$

Ocupación laboral de la madre:

- Empleado público/Funcionario $\quad 45,0 \%$

- Autónomo $\quad 5,0 \%$

- Trabajador por cuenta ajena $\quad 17,5 \%$

- Desempleado $\quad 12,5 \%$

- Jubilado 2,5\%

- Tareas domésticas $\quad 12,5 \%$

- Otro $\quad 5,0 \%$

Ingresos económicos familia:

- Menos de 707,7 euros 7,5\%

· Entre 707,8 y 1400 euros $\quad 10,0 \%$

- Entre 1401 y 2100 euros $\quad 32,5 \%$

- Entre 2101 y 2800 euros $\quad 17,5 \%$

· Más de 2800 euros 32,5\%

Posee Smartphone:

- Sí 97,5\%

- No $\quad 2,5 \%$

Posee Tablet:

· Sí $\quad 87,5 \%$

- No $\quad 12,5 \%$

Hijos con Smartphone propio:

- Sí $\quad 65,8 \%$

\section{Instrumento}

Se presenta un cuestionario inédito que forma parte de una investigación financiada con fondos nacionales.

El cuestionario como técnica cuantitativa de recogida de información, permite la obtención rápida y abundante de información mediante una serie de preguntas orales o escritas que debe responder un entrevistado con respecto a una o más variables a medir (Albert, 2007). En este caso se ha diseñado un cuestionario para ser autocumplimentado por las familias destinatarias. Igualmente, para facilitar la recogida y el análisis de la infor- mación, se subió el instrumento de evaluación diagnóstica a la plataforma LimeSurvey: https://encuestas.unican.es/encuestas/ index.php/342112?lang=es

La versión inicial del cuestionario "Smartphones y Tablets en familia" constaba de 105 preguntas, distribuidas en 8 bloques y dimensiones teóricas, algunas de ellas subdimensionadas, tal y como se muestra a continuación en la tabla 2. Para desarrollar el análisis psicométrico de la herramienta, se eliminaron las preguntas del bloque A "Datos identificativos" y las del bloque H “Necesidades Formativas" dado que estas preguntas son dicotómicas o de opción múltiple.

Tabla 2. Estructura inicial del cuestionario 
Bloque E: Rutinas y Hábitos

Bloque F: Normativa Familiar

Bloque G: Medicación Familiar

Boque H: Necesidades Formativas

Total, Ítems Cuestionario
Dimensión E1: Rutinas y Hábitos Familiares Smartphone 5

Dimensión E2: Rutinas y Hábitos Familiares Tablet 5

Dimensión E3: Rutinas y Hábitos Hijos Smartphone 6

Dimensión E4: Rutinas y Hábitos Hijos Tablet 2

Dimensión F1: Normativa Familiar Smartphone 3

Dimensión F2: Normativa Familiar Tablet 3

Dimensión F3: Normativa Hijos Smartphone 6

Dimensión F4: Normativa Hijos Tablet $\quad 4$

Dimensión G1: Mediación Familiar Hijos 7

Dimensión G2: Mediación Familiar Hijos con Smartphone 6

\section{Resultados}

Validez de contenido

El procedimiento de validación de contenido trata de evaluar el grado en el que los ítems del instrumento de recogida de información son representativos y relevantes en relación con el constructo que se desea medir. Como señalan Prieto y Delgado (2010), es la vía más usual para apreciar la calidad del contenido, especialmente en ámbitos educativos.

En la validación del instrumento que se presenta, participa- ron 8 expertos especialistas en diversos campos, pero todos ellos competentes en el ámbito educativo y tecnológico.

Junto con la versión del cuestionario online, se envió a cada experto la plantilla diseñada por Salcines-Talledo y González-Fernández (2016), que está conformada por preguntas cerradas para valorar con una escala tipo Likert, y preguntas abiertas, al final de cada bloque para que los expertos plasmasen sus sugerencias y recomendaciones.

A continuación, se presentan los resultados de la valoración por juicio de expertos de la validez de contenido del instrumento, agrupados por bloques de contenido.

Tabla 3. Valoración del cuestionario por los jueces expertos

\begin{tabular}{|c|c|c|}
\hline \multicolumn{3}{|c|}{ INTRODUCCIÓN } \\
\hline & Media & Desviación típica \\
\hline Claridad de los planteamientos & 3,50 &, 535 \\
\hline Adecuación a los destinatarios & 3,50 &, 535 \\
\hline Longitud del texto & 3,75 & 463 \\
\hline Calidad del contenido & 3,50 &, 535 \\
\hline \multicolumn{3}{|c|}{ REDACCIÓN DE LAS PREGUNTAS } \\
\hline & Media & Desviación típica \\
\hline Claridad de los planteamientos & 3,63 &, 518 \\
\hline Adecuación a los destinatarios & 3,50 & 756 \\
\hline Longitud del texto & 3,25 & 707 \\
\hline Calidad del contenido & 3,50 &, 535 \\
\hline \multicolumn{3}{|c|}{ NÚMERO Y ORDEN DE LAS PREGUNTAS } \\
\hline & Media & Desviación típica \\
\hline Orden de presentación lógico & 3,75 & ,463 \\
\hline Cantidad de preguntas & 3,38 & 744 \\
\hline \multicolumn{3}{|c|}{ BLOQUES Y DIMENSIONES DEL CUESTIONARIO } \\
\hline Organización & 3,63 &, 518 \\
\hline Cantidad & 3,25 & 886 \\
\hline Adecuación al contenido & 3,75 & 463 \\
\hline \multicolumn{3}{|c|}{ ESCALA DE RESPUESTA } \\
\hline Claridad & 3,75 & 463 \\
\hline Adecuación & 3,75 & 463 \\
\hline Cantidad & 3,75 & 463 \\
\hline Eficaces para discriminar los datos requeridos & 3,38 & 744 \\
\hline \multicolumn{3}{|c|}{ INSTRUCCIONES } \\
\hline Claridad & 3,38 & ,744 \\
\hline Adecuación & 3,38 &, 518 \\
\hline Cantidad & 3,50 &, 535 \\
\hline Calidad & 3,25 & 707 \\
\hline \multicolumn{3}{|c|}{ VALORACIÓN GENERAL DEL CUESTIONARIO } \\
\hline Validez del contenido del cuestionario & 3,75 & ,463 \\
\hline
\end{tabular}


Como se aprecia, en el primer bloque se hacen cuatro preguntas referentes a la introducción que se presenta con el cuestionario. La valoración general es positiva, con puntuaciones medias iguales o superiores a 3.5, teniendo en cuenta que la escala de valoración es de cuatro opciones de respuesta (siendo 1: Mala y 4: Excelente).

La valoración sobre redacción de las preguntas puede considerarse buena. La puntuación media más baja $(M=3,25$, d.t.= $0,707)$ aparece en el ítem sobre la longitud del texto de las preguntas. En la pregunta abierta del bloque, dos de los expertos coinciden al indicar que la redacción de algunas de las preguntas es confusa y demasiado extensa, de tal forma que en la versión final del cuestionario se ha simplificado y reducido la redacción de los ítems indicados.

Respecto al orden y número de las preguntas del cuestionario, los jueces ven adecuado el orden, pero consideran que podría reducirse el número de ítems. Con los análisis de fiabilidad que se presentan posteriormente, se han eliminado preguntas cuya supresión mejoraba la fiabilidad general de la escala.

En relación con los bloques y dimensiones del cuestionario, la pregunta cuya media presenta una puntuación menor $(M=$ 3,25 , d.t. $=0,886$ ) es la relativa al número de dimensiones. De igual modo, los análisis de fiabilidad han permitido fusionar dos dimensiones en la versión final.

Los evaluadores consideran que la escala del cuestionario con cuatro opciones de respuesta es la adecuada y eficaz para conseguir los objetivos propuestos. Por lo tanto, la escala se mantuvo igual.
En lo referente a las instrucciones que acompañan al cuestionario encontramos valoraciones medias iguales o superiores a 3,25. En la pregunta abierta del bloque, uno de los jueces indicó que algunos comentarios podrían simplificarse.

Finalmente, cabe destacar que la valoración global del cuestionario por parte de los jueces es muy positiva $(M=3,75$, d.t.= $0,463)$.

\section{Fiabilidad del cuestionario mediante análisis de la consistencia interna}

La fiabilidad expresa el grado de precisión, estabilidad y consistencia que manifiesta un cuestionario como instrumento de medida. En este caso se determina la fiabilidad o consistencia interna mediante la prueba Alfa de Cronbach, cuyos valores oscilan entre 0 y 1 . Se consideran valores altos cuando superan 0,71 y muy altos cuando sobrepasan 0,91 (Mateo, 2004).

Para calcular la fiabilidad del cuestionario se empleó el programa estadístico SPSS 22.0. Una vez eliminadas las preguntas del bloque identificativo y las preguntas del bloque $\mathrm{H}$ se procedió a calcular el Alfa de Cronbach para cada uno de los bloques que componen el cuestionario, debido a que se han tratado como escalas independientes dentro de una misma herramienta como consecuencia de su propia entidad teórica individual. A continuación, presentamos los datos de la fiabilidad inicial, y la fiabilidad posterior, después de eliminar los ítems cuya supresión mejoraba la fiabilidad global de la escala y fusionar los bloques B y C, al comprobar que tienen una mayor consistencia interna global.

Tabla 4. Resumen de los coeficientes de fiabilidad por el método Alfa de Cronbach

\begin{tabular}{|c|c|c|c|c|c|c|}
\hline \multirow[t]{2}{*}{ BLOQUES INICIALES } & \multicolumn{2}{|c|}{$\begin{array}{l}\text { DATOS ESCALAS } \\
\text { INICIALES }\end{array}$} & \multirow[t]{2}{*}{ BLOQUES DESPUÉS DE ANÁLISIS } & \multirow{2}{*}{$\begin{array}{l}\text { ÍTEMS QUE } \\
\text { ELIMINAR }\end{array}$} & \multicolumn{2}{|c|}{$\begin{array}{c}\text { DATOS ESCALAS } \\
\text { DESPUÉS DE ANÁLISIS }\end{array}$} \\
\hline & № ÍTEMS & ALFA & & & № ÍTEMS & ALFA \\
\hline $\begin{array}{l}\text { Bloque B: Conocimiento } \\
\text { General }\end{array}$ & 3 & ,334 & \multirow{2}{*}{ Bloque B: Conocimiento } & \multirow{2}{*}{ B_3 } & \multirow{2}{*}{6} & \multirow{2}{*}{ 709 } \\
\hline $\begin{array}{l}\text { Bloque C: Conocimiento De } \\
\text { Dispositivos Móviles }\end{array}$ & 5 & 199 & & & & \\
\hline $\begin{array}{l}\text { Bloque D: Comunicación } \\
\text { Familiar }\end{array}$ & 25 & 879 & Bloque C: Comunicación Familiar & $\begin{array}{l}\text { D1_4 } \\
\text { D2_4 } \\
\text { D4_1 } \\
\text { D5_3 } \\
\text { D6_1 } \\
\text { D6_3 }\end{array}$ & 19 & 890 \\
\hline $\begin{array}{l}\text { Bloque E: Rutinas y } \\
\text { Hábitos }\end{array}$ & 18 & ,767 & Bloque D: Rutinas y Hábitos & $\begin{array}{l}\text { E2_2 } \\
\text { E2_4 } \\
\text { E2_5 }\end{array}$ & 15 & 816 \\
\hline $\begin{array}{l}\text { Bloque F: Normativa } \\
\text { Familiar }\end{array}$ & 16 & ,920 & Bloque E: Normativa Familiar & $\begin{array}{l}\text { F3_1 } \\
\text { F3_5 } \\
\text { F4_1 }\end{array}$ & 13 & ,931 \\
\hline $\begin{array}{l}\text { Bloque G: Medicación } \\
\text { Familiar }\end{array}$ & 13 & 866 & Bloque F: Medicación Familiar & G1_7 & 12 & 891 \\
\hline
\end{tabular}

Tabla 5. Estructura final del cuestionario

Finalmente, el cuestionario está compuesto por 7 bloques y 91 ítems tal y como se recoge en la tabla 5. Todos los valores de Alfa de Cronbach finales son superiores a 0,7, lo que indica una alta fiabilidad de la herramienta de evaluación diagnóstica diseñada.

\section{BLOQUES/DIMENSIONES}

Bloque A: Datos Identificativos

Bloque B: Conocimiento

№ ÍTEMS

Bloque C: Comunicación Familiar

Bloque D: Rutinas y Hábitos

Bloque E: Normativa Familiar

Bloque F: Medicación Familiar

Boque G: Necesidades Formativas

TOTAL, ÍTEMS CUESTIONARIO

\section{3}

6

19

15

13

12

3

91 
Resultados destacables tras la aplicación piloto

A continuación, se presentan algunos resultados destacables tras analizar la aplicación piloto, que pueden indicar hacia dónde podrían ir los resultados de los datos que se recojan con la aplicación definitiva del cuestionario en nivel nacional, y nos muestran el adecuado comportamiento del instrumento.

En primer lugar, respecto al uso mayoritario que las familias realizan de los dispositivos tecnológicos, tal y como aparece en la tabla 6, destacan el Smartphone (75\%) y la Tablet $(77,5 \%)$ como los dispositivos más utilizados con fines recreativos, mientras que el ordenador portátil (65\%), seguido del ordenador de sobremesa $(47,5 \%)$, se presentan como los dispositivos preferidos para realizar un uso educativo.

Tabla 6. Tipos de uso de los dispositivos tecnológicos

\section{TIPOS DE USO DE LOS DISPOSITIVOS}

\section{PORCENTAJE DE USO}

\section{Tablet:}

- Uso mayoritariamente recreativo

Uso mayoritariamente educativo

- No dispone de dicho dispositivo

- No sabe/ No contesta
$77,5 \%$
$7,5 \%$
$10,0 \%$
$5,0 \%$
$25,0 \%$
$65,0 \%$
$7,5 \%$
$2,5 \%$
$17,5 \%$
$47,5 \%$
$25,0 \%$
$10,0 \%$
$32,5 \%$
$10,0 \%$
$37,5 \%$
$20,0 \%$
$75,0 \%$
$10,0 \%$
$2,5 \%$

Portátil:

Uso mayoritariamente recreativo

Uso mayoritariamente educativo

No dispone de dicho dispositivo

- No sabe/ No contesta

Ordenador de sobremesa:

- Uso mayoritariamente recreativo

Uso mayoritariamente educativo

No dispone de dicho dispositivo

No sabe/ No contesta

eBook:

- Uso mayoritariamente recreativo

Uso mayoritariamente educativo

No dispone de dicho dispositivo

- No sabe/ No contesta

Smartphone:

- Uso mayoritariamente recreativo

- Uso mayoritariamente educativo

No sabe/ No contesta

En relación con el conocimiento que los padres poseen sobre los dispositivos móviles, destacan valores medios inferiores al punto medio de la escala 2,5 (escala de 1 a 4; siendo 1: Totalmente en desacuerdo y 4: Totalmente de acuerdo). Concretamente, dichos valores aparecen en los ítems relativos al conocimiento sobre la jerga tecnológica que emplean los jóvenes $(M=2,27$, d.t.= $0,871)$, y al conocimiento sobre la normativa legal que regula la protección de la ciudadanía ante los medios tecnológicos $(M=$ $2,45$, d.t. $=0,860)$. En contraposición, los progenitores presentan una puntuación muy elevada en la pregunta relativa a los riesgos que pueden surgir cuando los menores realizan un uso inadecuado de los dispositivos móviles $(M=3,58$, d.t.=0,758).

Respecto a las preguntas del cuestionario concernientes al bloque de "Comunicación", destaca la presencia de puntuaciones medias bajas. Los ítems que presentan las puntuaciones superiores son: "He podido contactar con familiares con los que apenas tenía contacto" $(M=2,97$, d.t.= 0,866); “Mi/s hijo/s pierden la atención (cuando le hablas, cuando hace deberes, etc.) por estar utilizando el Smartphone de los padres" $(M=2,88$, d.t. $=0,907)$ $\mathrm{y} ;$ " Mi/s hijo/s a menudo pierden la atención en la conversacio-

nes cara a cara familiares por estar usando la Tablet" $(M=2,72$, d.t. $=0,996)$. Por el contrario, entre los ítems cuyas puntuaciones son inferiores, destacan: "Me comunico durante más tiempo con mi pareja a través del Smartphone que en persona" $(M=1,65$, d.t. $=0,797) \mathrm{y}$; "Ha aumentado la variedad de temas de comunicación con mi pareja gracias al uso del Smartphone" $(M=1,72$, d.t. $=0,678)$.

Los ítems que conforman el bloque de "Rutinas y Hábitos" con puntaciones superiores a la media de la escala son: "En los momentos de ocio familiar (pasear, charlar, ir a la montaña, etc.) a menudo me encuentro pendiente del Smartphone" $(M=2,56$, d.t. $=0,882)$; "Suelo volver a casa, si he olvidado el Smartphone" $(M=2,92$, d.t. $=0,850) \mathrm{y}$; "Dejamos el Smartphone a nuestros hijos para que realicen consultas académicas en internet" $(M=2,88$, d.t. $=0,900)$;. Mientras que las preguntas destacables por tener puntuaciones inferiores son: “Cuando me voy a la cama a descansar, sigo pendiente del Smartphone" $(M=1,95$, d.t.= 0,916) y; "Mi hijo suele utilizar mi móvil todos los días" $(M=1,82$, d.t.= 0,983).

Los resultados del bloque relativo a la normativa familiar presentan puntuaciones medias muy elevadas, destacando los ítems relacionados con el conocimiento del tiempo y finalidad de uso que los menores realizan de los dispositivos móviles con medias superiores a 3,00.

El bloque "Mediación familiar", en general presenta puntuaciones medias elevadas, destacando los ítems: "Sensibilizo a mi/s hijo/s sobre los riesgos y peligros que puede conllevar un uso inadecuado de los dispositivos móviles: publicidad engañosa, suplantación de personalidad, difusión de virus, compras onli$n e^{\prime \prime}(\mathrm{M}=3,33$, d.t.=0,736) y; “Siento que la educación/valores que le estoy transmitiendo a mi/s hijo/s en relación a los dispositivos móviles es la más adecuada" ( $\mathrm{M}=3,00$, d.t.=0,577).

Finalmente, cabe destacar, que, a las familias participantes en la aplicación piloto del cuestionario, no les interesa recibir una formación que les permita ampliar su conocimiento sobre el uso de los dispositivos móviles en el contexto familiar.

\section{Discusión y conclusiones}

$\mathrm{Al}$ inicio de este artículo se planteaba como objetivo del mismo ofrecer los resultados del proceso de validación y aplicación piloto de un instrumento de evaluación diagnóstica sobre el impacto de los dispositivos móviles en el contexto familiar. En este sentido, las escalas analizadas se configuran como herramientas que posibilitan el avance en el conocimiento de las relaciones que se establecen entre las familias y estos dispositivos móviles, muestra de ello han sido los adecuados parámetros obtenidos en el análisis psicométrico de las mismas.

Si se reflexiona sobre los primeros hallazgos obtenidos en la aplicación piloto de las escalas, resulta paradójico comprobar cómo los padres y madres son conscientes de los riesgos que conlleva el uso de los dispositivos móviles. Sin embargo, desconocen la normativa que regula las relaciones de los usuarios con estos medios y el vocabulario específico empleado por sus hijos en el uso de Smartphones y Tablets. Parece urgente que este hecho se confirme en el estudio posterior con una muestra mayor, pues si esta es la realidad de las familias españolas, se evidencia una brecha digital entre generaciones mucho mayor de la que se pudiera prever a priori. Al mismo tiempo, mostraría la necesidad de tomar medidas desde diferentes esferas para paliar un posible problema de salud pública debido a la vulnerabilidad que podrían sufrir los menores si sus padres no se encuentran lo suficientemente capacitados para hacer frente a dichos riesgos (Haughton, Aiken y Cheevers, 2015; Radesky y Christakis, 2016), 
a pesar de que afirmar saber qué hacen sus hijos cuando interactúan con estos dispositivos y de las medidas que manifiestan adoptar con ellos.

Esta idea se sigue poniendo de manifiesto en lo que concierne a la comunicación que se genera cuando se usan estos dispositivos móviles, provocando que los hijos pierdan la atención respecto a otras tareas que están realizando, o cuando se mantiene una comunicación face to face con otras personas, situaciones semejantes se han descrito en los trabajos de Hiniker et al., (2015), Hiniker, Schoenebeck y Kientz (2016) y/o McDaniel y Coyne (2016). Las familias también afirman que estos medios emergentes han provocado un incremento de las relaciones con otros familiares, mientras que los mismos no han conseguido aumentar la comunicación con la pareja ni los temas de conversación que suelen tratar.

Los resultados han revelado una dependencia de los padres hacia el móvil, teniendo que regresar a casa si se les ha olvidado el Smartphone e interrumpiendo los momentos de ocio familiar por “estar pendientes" del dispositivo. Sobre esta cuestión resultan significativas las investigaciones de Marsh et al. (2015) y Stephen et al. (2013), quienes destacan la importancia del uso parental del dispositivo móvil cuando se encuentran en presencia de sus hijos o las realizadas por Harmon y Mazmanian (2013), Sharaievska y Stodolska (2016) y Yu (2015) en relación al funcionamiento familiar y la gestión de los dispositivos móviles por parte de los propios padres, que evidencian la falta de una vida familiar cuando "se interponen", fundamentalmente, los Smartphones.

Todas estas manifestaciones podrían apuntar hacia una dualidad en la relación familias y dispositivos móviles, una aceptación de carencias, pero que no requiere de subsanación por parte de las familias porque "esto es lo que hay", "así es la sociedad hiperconectada" o "todas las familias tenemos el mismo problema con nuestros hijos". Esta falta de reflexión crítica sobre el estilo parental que se está ejerciendo en las familias en torno a la educación de estos medios emergentes en la muestra participante, se confirma con la falta de interés por una formación en torno a una educación mediática de sus hijos e hijas.

El análisis de los datos que se obtengan de la aplicación del cuestionario en una muestra mayor indicará si se trata de una tendencia generalizada en todo el territorio nacional. La confirmación o no de estos términos posibilitará generar un diagnóstico real del impacto de los dispositivos móviles en las familias españolas.

\section{Referencias Bibliográficas}

Albert, M.J. (2007). La investigación educativa: claves teóricas. Madrid: McGraw-Hill.

Álvarez-García, D., Dobarro, A. y Núñez, J.C. (2015). Validez y fiabilidad del Cuestionario de cibervictimización en estudiantes de Secundaria. Aula Abierta, 43, 32-38.

Bianchi, A. y Phillips, J.G. (2005). Psychological predictors of problem mobile phone use. Cyberpsychology \& Behavior: The Impact of the Internet, Multimedia and Virtual Reality on Behavior and Society, 8(1), 39-51.

Billieux, J., Van der Linden, M. y Rochat, L. (2008). The role of impulsivity in actual and problematic use of the mobile phone. Applied Cognitive Psychology, 22(9), 1195-1210.

De la Villa, M. y Suárez, C. (2016). Factores de riesgo en el uso problemático de Internet y del teléfono móvil en adolescentes españoles. Revista Iberoamericana de Psicología y Salud, 7, 69-78.

Digital in 2017 (2017). We Are Social: global overview. Recuperado de: https://wearesocial.com/special-reports/digital-in-2017-global-overview
Dryāgan, (2010). Usos y abuso de las tecnologías. Recuperado de: http://www.slideshare.net/kajinoinazuma/uso-yabusodelastecnologias

Fombona, J. y Roza, P. (2016). Uso de los dispositivos móviles en educación infantil. Edmetic, 5(2), 158-181.

Fundación Telefónica (2017). La Sociedad de la Información en España 2016. Madrid: Fundación Telefónica.

Gil Pascual, J.A. (2016). Técnicas e instrumentos para la recogida de información. Madrid: UNED.

Harmon, E. y Mazmanian, M. (2013). Stories of the smartphone in everyday discourse: Conflict, tension \& instability. En Proceedings of the SIGCHI conference on human factors in computing systems (pp. 1051-1060). doi: http://dx.doi.org/ $10.1145 / 2470654.2466134$.

Haughton, C., Aiken, M. y Cheevers, C. (2015). Cyber babies: The impact of emerging technology on the developing infant. Psychology, 5(9), 504-518. doi: http://dx.doi.org/10.17265/21595542/2015.09.002.

Henríquez, P., González, C. y Organista, J. (2014). Clasificación de perfiles de uso de smartphones en estudiantes y docentes de la Universidad Autónoma de Baja California, México. Revista Complutense de Educación, 25(2), 245-270.

Hiniker, A., Schoenebeck, S.Y. y Kientz, J.A. (2016). Not at the dinner table: Parents' and children's perspectives on family technology rules. En Proceedings of the 19th ACM conference on computer-supported cooperative work E social computing (pp. 1376-1389). doi: http://dx.doi.org/10.1145/281804.28199940.

Hiniker, A., Sobel, K., Suh, H., Sung, Y. C., Lee, C.P. y Kientz, J.A. (2015). Texting while Parenting: How adults use mobile phones while caring for children at the playground. En Proceedings of the 33rd annual ACM conference on human factors in computing systems (pp. 727-736). doi: http://dx.doi. org/10.1145/ 2702123.2702199.

Informe Ditrendia (2017). Informe Mobile en España y en el Mundo 2017. Ditrendia.

Instituto Nacional de Estadística (2017). Encuesta sobre Equipamiento y Uso de Tecnologías de Información y Comunicación en los Hogares. Recuperado de: http://www.ine.es/jaxi/Datos. htm?path=/t25/p450/base 2011/a2017/10/\&file=01004.px

Jenaro, C., Flores, N., Gómez-Vela, M., González-Gil, F. y Caba1lo, C. (2007). Problematic internet and cell-phone use: Psychological, behavioral, and health correlates. Addiction Research E Theory, 15(3), 309-320.

Kawasaki, N., Tanei, S., Ogata, F., Burapadaja, S., Loetkham, C., Nakamura, T. y Tanada, S. (2006). Survey on Cellular Phone Usage on Students in Thailand. Journal of Physiological Anthropology, 25(6), 377-382.

Lepicnik, J. y Samec, P. (2013). Uso de tecnologías en el entorno familiar en niños de cuatro años de Eslovenia. Comunicar, 20(40), 119-126.

López-Sánchez, C. y García del Castillo, J.A. (2017). La familia como mediadora ante la brecha digital: repercusión en la autoridad. Revista Colombiana de Ciencias Sociales, 8(1), 108-124.

Martínez, M., Enciso, R. y González Castillo, S. (2015). Impacto del uso de la tecnología móvil en el comportamiento de los niños en las relaciones interpersonales. Revista Educateconciencia, 5(6), 67-80.

Mateo, J. (2004). La investigación ex post-facto. En R. Bisquerra-Alzina (coord.). Metodología de la investigación educativa (pp. 195-230). Madrid: La Muralla.

McDaniel, B. T. y Coyne, S. M. (2016). Technology interference in the parenting of young children: Implications for mothers' perceptions of coparenting. The Social Science Journal, 53(4), 435-443. doi: http://dx.doi.org/10.1016/j.soscij.2016.04.010. 
Marsh J., Plowman L., Yamada-Rice D., Bishop J. C., Lahmar J., Scott F., et al. (2015). Exploring Play and Creativity in Pre-Schoolers' Use of Apps: Final Project Report. Recuperado de: https://goo.gl/Fs9X3X.

Merino, E., Cabello, J. y Merino, E. (2017). El teléfono móvil y los estudiantes universitarios: una aproximación a usos, conductas y percepciones. Píxel-Bit. Revista de Medios y Educación, 51, 81-96.

Ortega, F., González, B. y Pérez, M.E. (2015). Audiencias en revolución, usos y consumos de las aplicaciones de los medios de comunicación en tabletas y teléfonos inteligentes. Revista Latina de Comunicación Social, 70, 627-651.

Pedrero, E., Rodríguez, M. y Ruiz, J. (2012). Adicción o abuso del teléfono móvil. Adicciones, 24(2), 139-152. Recuperado de: http://www.redalyc.org/articulo.oa?Id=289122912007

Prensky, M. (2009). Homo sapiens digital: From immigrants and digital natives to digital wisdom. Innovate, 5(3). Recuperado de: http://www.innovateonline.info/pdf/vol5 issue3/H. Sapiens Digital- From_Digital_Immigrants and Digital_Natives to Digital Wisdom.pdf

Prieto, G. y Delgado, A.R. (2010). Fiabilidad y validez. Papeles del Psicólogo, 31(1), 67-74.

Radesky, J.S. y Christakis, D.A. (2016). Increased screen time: Implications for early childhood development and behavior. Pediatric Clinics of North America, 63(5), 827-839. doi: http:// dx.doi.org/10.1016/j.pcl.2016.06.006.

Ramos, D. y Ortega-Mohedano, F. (2017). La revolución en los hábitos de uso y consumo de vídeo en teléfonos inteligentes entre usuarios Millenials, la encrucijada revelada. Revista Latina de Comunicación Social, 72, 704-718.

Rodríguez-Gómez, D., Castro, D. y Meneses, J. (2018). Usos problemáticos de las TIC entre jóvenes en su vida personal y escolar. Comunicar, 56, 91-100.

Salcines-Talledo, I. y González-Fernández, N. (2016). Diseño y Validación del Cuestionario Smartphone y Universidad. Visión del Profesorado (SUOL). Revista Complutense de Educación, 27(2), 603-632.
Salcines-Talledo, I., y González-Fernández, N. (2015). Los Smartphones en Educación Superior. Diseño y validación de dos instrumentos de recogida de información sobre la visión del alumnado. REOP. Revista Española de Orientación y Psicopedagogía, 26(3), 96-120.

Sharaievska, I. y Stodolska, M. (2016). Family satisfaction and social networking leisure. Leisure Studies, 1-13. doi: http://dx. doi.org/10.1080/02614367.2016.1141974

Stein, C.H., Lawrence, A.O., y Greenberg, S.C. (2016). Understanding Young Adults' Reports of Contact with their Parents in a Digital World: Psychological and Familial Relationship Factors. J Child Fam Stud, 25, 1802-1814.

Stephen C., Stevenson O. y Adey C. (2013). Young children engaging with technologies at home: the influence of family context. Journal of Early Childhood Research, 11, 149-164. doi: 10.1177/1476718X12466215.

Symons, K., Ponnet, K. Emmery, K., Walrave, M., y Heirman, W. (2017). Parental Knowledge of Adolescents' Online Content and Contact Risks. J Youth Adolescence, 46, 401-416

Tanis, M., Louw, M., y Buijzen, M. (2017). From empty nest to Social Networking Site: What happens in cyberspace when children are launched from the parental home? Computers in Human Behavior, 68, 56-63.

Toda, M., Monden, K., Kubo, K., y Morimoto, K. (2006). Mobile Phone Dependence and Health-Related Lifestyle of University Students. Social Behavior and Personality: An International Journal, 34(10), 1277-1284.

Tsai, T-H, Chang, H-T, y Ho, Y-L (2016). Perceptions of a Specific Family Communication Application among Grandparents and Grandchildren: An Extension of the Technology Acceptance Model. Plos One. Recuperado de: https://www.ncbi. nlm.nih.gov/pmc/articles/PMC4896451/

Yang, Y-S., Yen, J-Y., Ko, C-H., Cheng, C-P. y Yen, C-F. (2010). The association between problematic cellular phone use and risky behaviors and low self-esteem among Taiwanese adolescents. BMC Public Health, 10, 217.

$\mathrm{Yu}$, X. (2015). Smartphone usage and family vacation experiences (Unpublished doctoral dissertation). Lafayette: Purdue University. Recuperado de: https://goo.gl/dMQCsk. 